\title{
MicroRNA-125b suppresses the proliferation and osteogenic differentiation of human bone marrow-derived mesenchymal stem cells
}

\author{
SHI CHEN ${ }^{1 *}$, LIU YANG ${ }^{1 *}$, QIANG JIE ${ }^{1}$, YAN-SHUI LIN ${ }^{2}$, GUO-LIN MENG ${ }^{1}$, \\ JIN-ZHU FAN ${ }^{1}$, JIN-KANG ZHANG ${ }^{1}$, JING FAN ${ }^{1}$, ZHUO-JING LUO $^{1}$ and JIAN LIU ${ }^{1}$ \\ ${ }^{1}$ Department of Orthopaedics, Xijing Hospital, Fourth Military Medical University, Xi'an, Shaanxi 710032; \\ ${ }^{2}$ Department of Orthopaedics, First Affiliated Hospital, Chengdu Medical College, Chengdu, Sichuan 610500, P.R. China
}

Received June 30, 2013; Accepted February 19, 2014

DOI: $10.3892 / \mathrm{mmr} .2014 .2024$

\begin{abstract}
The regressive biological function of human bone marrow-derived mesenchymal stem cells (hBMSCs) is one of the key factors resulting in the decrease of bone mass in senile osteoporosis. MicroRNAs (miRs) are non-coding small RNAs involved in various gene regulation processes. Whether any $\mathrm{miR}(\mathrm{s})$ are involved in the progression of osteoporosis by regulating the biological function of hBMSCs remains to be elucidated. The present study aimed to compare the expression levels of miR-125b in hBMSCs derived from senile osteoporotic patients with that of control (normal) subjects. A significantly upregulated expression of miR-125b in osteoporotic hBMSCs was detected. To elucidate the biological function of miR-125b in senile osteoporosis, the effects of miR-125b expression on proliferation and osteogenic differentiation of hBMSCs were assessed using gain- and loss-of-function studies. It was evident that the overexpression of a miR-125b mimic was able to suppress the proliferative and osteogenic differentiation of senile hBMSCs. In contrast, repression of the function of miR-125b by transfection of an miR-125b inhibitor promoted the proliferation and osteogenic differentiation of hBMSCs. Furthermore, the potential target gene of miR-125b, osterix $(O s x)$, was examined. The results of the present study strongly suggested that miR-125b may regulate osteogenic differentiation of hBMSCs through the modulation of Osx expression.
\end{abstract}

Correspondence to: Professor Jian Liu or Professor Zhuo-Jing Luo, Department of Orthopaedics, Xi-jing Hospital, Fourth Military Medical University, 15 Changle West Road, Xi'an, Shaanxi 710032, P.R. China

E-mail: ljreny@fmmu.edu.cn

E-mail: zjluo@fmmu.edu.cn

${ }^{*}$ Contributed equally

Key words: microRNA-125b, osteoporosis, bone marrow-derived mesenchymal stem cells, osterix

\section{Introduction}

Bone marrow mesenchymal stem cells (BMSCs) are multipotent progenitor cells with the capacity of differentiating into osteoblasts, chondrocytes, adipocytes and myoblasts (1-3). Previous studies have shown that the multi-differentiation processes of BMSCs are tightly regulated by various factors. Recently, microRNAs (miRNAs or miRs) have been reported to have crucial roles in the osteogenic differentiation of BMSCs. For example, miR-204 and -211 inhibit osteogenesis of hBMSCs by acting as endogenous negative regulators of Runt-related transcription factor-2 (Runx-2) (4). miR-138 suppresses osteogenic differentiation of hBMSCs by repressing the expression of focal adhesion kinase (FAK), as well as its downstream targets (5). Furthermore, miR-637 significantly suppresses osteogenic differentiation in hBMSCs through direct repression of osterix $(O s x)$ expression (6). Previous studies have reported that miR-125b is a crucial post-transcriptional regulator of genes that are involved in cell proliferation or differentiation processes of various cell lineages (7-10). However, the role of miR-125b in hBMSCs and its downstream target genes have not been fully elucidated.

Osteoporosis, a common disease of the skeletal system, is characterized by the loss of bone mass due to an imbalance in relative bone secretion versus bone resorption (11). Disruptive bone metabolism or levels of bone secretion which are disproportionate to resorption result in decreased bone mass or osteoporosis. Excessive accumulation of adipocytes is frequently observed in the bone marrow of patients with senile osteoporosis, which may suggest an imbalanced differentiation of BMSCs into osteoblasts or adipocytes (12). The osteogenic differentiation of BMSCs is a well-coordinated process and is regulated by key transcription factors, including Runx-2 and Osx $(13,14)$. However, whether miR-125b has a regulatory role in the proliferation and osteogenic differentiation of hBMSCs remains to be elucidated. In this context, the present study aimed to assess the impact of miR-125b and Osx expression on the proliferation and osteogenic differentiation of hBMSCs derived from senile osteoporotic patients. 


\section{Materials and methods}

Bone marrow donors. Written informed consent was obtained from all participating subjects and the present study was approved by the ethics committee of the Fourth Military Medical University (Xi'an, China). Bone marrow aspirates of $5-10 \mathrm{ml}$ from the iliac crest were obtained from five normal donors and four elderly osteoporotic patients who had undergone an iliac bone graft procedure during surgery. Normal donors had a T-score of >-1 standard deviation (SD) and no history of bone fractures. A standardized clinical evaluation was performed to exclude possible comorbidities. Exclusion criteria included premature menopause, the presence of a disease and the use of drugs that are able to affect bone or calcium and phosphorus metabolism. Osteoporosis was diagnosed in accordance with the World Health Organization parameters, with a T-score of $<-2.5 \mathrm{SD}$. Calcium, phosphate, parathyroid hormone, carboxy-terminal telopeptide of type I collagen, alkaline phosphatase (ALP), 25-hydroxyvitamin D levels and the urinary calcium excretion rate were measured in the serum of all elderly patients with osteoporotic fractures to exclude any secondary causes of osteoporosis.

Isolation and culture of hBMSCs. hBMSCs were isolated and cultured as previously described (15). Bone marrow aspirates of $5-10 \mathrm{ml}$ were placed in tubes containing heparin (100 U/l) and were mixed with isochoric phosphate-buffered saline (PBS). The cell suspension was mixed with an equal volume $(1.073 \mathrm{~g} / \mathrm{ml})$ of Percoll solution (Pharmacia, Uppsala, Sweden) in $15 \mathrm{ml}$ conical tubes (Nunc A/S, Roskilde, Denmark) and centrifuged (L500 Centrifuge; Xiangyi Centrifuge Instrument Co., Ltd., Hunan, China) at $644.8 \mathrm{x}$ g for $30 \mathrm{~min}$. Mononuclear cells were collected from the middle layer and interface, diluted with two volumes of PBS and collected by centrifugation at $161.2 \mathrm{x}$ g. The cells were resuspended in complete culture medium (low-glucose Dulbecco's modified Eagle medium with $10 \%$ fetal bovine serum, $100 \mathrm{U} / \mathrm{ml}$ penicillin, $100 \mathrm{mg} / \mathrm{ml}$ streptomycin and 2 mM L-glutamine; Hyclone, Logan, UT, USA). The cells were seeded at 4,500 cells $/ \mathrm{cm}^{2}$ in $25 \mathrm{~cm}^{2}$ culture flasks (Nunc A/S) and were incubated at $37^{\circ} \mathrm{C}$ in $5 \% \mathrm{CO}_{2}$ with $95 \%$ humidity. After three days of incubation, the cultured medium and non-adherent cells were discarded, adherent cells were washed twice with PBS and resuspended in fresh culture medium. After 10-14 days of culture, the cells were harvested using $0.25 \%$ trypsin and $1 \mathrm{mM}$ EDTA (Gibco, Grand Island, NY, USA) and were replated at $10^{4}$ cells $/ \mathrm{cm}^{2}$ in $25 \mathrm{~cm}^{2}$ culture flasks (Nunc A/S) to expand the cells through successive passages.

For osteogenic differentiation, hBMSCs were seeded at $10^{4}$ cells $/ \mathrm{cm}^{2}$ in $25 \mathrm{~cm}^{2}$ culture flasks (for RNA isolation; Nunc $A / S$ ) or in six-well plates (for staining). Cells were grown to $45-75 \%$ confluency over $24-48 \mathrm{~h}$ in standard growth medium. On every third day, the medium was replaced with osteogenic differentiation medium $(10 \%$ fetal bovine serum, Hyclone; $100 \mathrm{nM}$ dexamethasone, $45 \mathrm{mM}$ L-ascorbic acid and $10 \mathrm{mM} \beta$-glycerophosphate; Sigma, St. Louis, MO, USA). Samples were stained or harvested for RNA isolation on the 14th day of differentiation.

Transfection of miR-125b mimic and inhibitor. The miR-125b mimic, miR-125b inhibitor and a non-specific negative control (NC) were designed and synthesized by Invitrogen Life Technologies (Shanghai, China). The sequences included: miR-125b mimic, 5'-UCCCUGAGACCCUAACUUGUGAA CAAGUUAGGGUCUCAGGGAUU-3'; miR-125b inhibitor, 5'-UCACAAGUUAGGGUCUCAGGGA-3' and NC, 5'-CAG UACUUUUGUGUAGUACAA-3'.

The negative control, a random sequenced anti-miRNA molecule based on the miRNAs in C. elegans, has been extensively compared to the human, mouse and rat genome sequences and miRNA sequences by the Basic Logarithmic Alignment Search Tool for nucleotides (BLASTn; National Library of Medicine, Bethesda, MD, USA) and validated to not produce identifiable effects on the known miRNA functions.

Cells were transfected using Lipofectamine 2000 reagent (Invitrogen, Carlsbad, CA, USA). The hBMSCs were plated in six-well plates (Nunc A/S) to $45 \%$ confluence. In addition, $20 \mu \mathrm{M}$ miR-125b mimic or a miR-125b inhibitor were mixed with Lipofectamine 2000, which was prepared according to the manufacturer's instructions. The final concentrations of miR-125b mimic or miR-125b inhibitor and the negative control were adjusted to $45 \mathrm{nM}$. The culture conditions were conducive and the survival rate of hBMSCs was up to 90\% following transfection. After the transfection $(4 \mathrm{~h})$, the medium was replaced with osteogenic differentiation medium to induce osteoblast differentiation.

RNA extraction. Total RNA was isolated from cultured cells using TRIzol ${ }^{\circledR}$ reagent (Invitrogen), according to the manufacturer's instructions. The integrity and purity of total RNA was verified spectrophotometrically and by gel-electrophoresis on formaldehyde denaturation gel.

Quantitative polymerase chain reaction ( $q P C R)$. Gene expression levels were measured by the iQ5 real-time PCR detection system (Bio-Rad, Hercules, CA, USA). microRNAs were prepared with an All-in-One ${ }^{\mathrm{TM}}$ miRNA qRT-PCR detection kit (AOMD-Q020; GeneCopoeia, Inc., Rockville, MD, USA) according to the manufacturer's instructions. Briefly, the extracted RNA was reverse-transcribed in the presence of a poly-A polymerase with an oligo-dT adaptor. qPCR was then performed with SYBR ${ }^{\circledR}$ Green (Takara, Shiga, Japan) detection with a forward primer for the mature miRNA sequence and a universal adaptor reverse primer. RNA was reverse-transcribed using the All-in-One ${ }^{\mathrm{TM}}$ miRNA qPCR Primer (GeneCopoeia, Inc.).

The sequence of the F-Primer for human snRNA U6 was: TCGTGAAGCGTTCCATATTTTTAA. The sequence of the F-Primer for Homo sapiens (hsa)-miR-125b was: CCCTGAGACCCTAACTTGTGAAA and the sequence of the Mature1_Seq for hsa-miR-125b was: UCCCUGAGACCCUAACUUGUGA. The expression of Runx-2, ALP, Osteocalcin (OC), collagen type I $\alpha 1$ (COLI $\alpha 1)$ and $O s x$ were quantified by qPCR using SYBR ${ }^{\circledR}$ Green assays (Takara) and GAPDH was used as an internal control.

PCR primers included: Runx-2 forward, CCACCACTCAC TACCACACCTA and reverse, CCTGACGAAGTGCC ATAGTAGA; ALP forward, GCATAACATCAGGGACAT TGAC and reverse, TGGCTTTCTCGTCACTCTCAT; $O C$ forward, CTCACACTCCTCGCCCTATT and reverse, CG 
Table I. Clinical characteristics of the normal donors and patients with osteoporosis.

\begin{tabular}{lccr}
\hline Groups & Age (years) & Gender & BMD t-score \\
\hline Osteoporotic patient & 76 & Female & -2.7 SD \\
Osteoporotic patient & 82 & Male & -2.8 SD \\
Osteoporotic patient & 82 & Female & -3.0 SD \\
Osteoporotic patient & 88 & Female & -2.6 SD \\
Control & 19 & Female & 0.8 SD \\
Control & 28 & Male & 0.6 SD \\
Control & 40 & Female & -0.8 SD \\
Control & 44 & Male & -0.4 SD \\
Control & 38 & Male & 0.7 SD \\
\hline
\end{tabular}

Bone densitometry values in osteoporotic patients were lower than those in the normal donors. All subjects were of Chinese origin. BMD, bone mineral density.

CTGGGTCTCTTCACTAC; COL1 1 forward, AGGGCT CCAACGAGATCGAGATCCG and reverse, TACAG GAAGCAGACAGGGCCAACG; Os $x$ forward, ACCTACCC ATCTGACTTTGCTC and reverse, CTGCCCACTATTTCC CACTG; GAPDH forward, GGAGACAACCTGGTCCTCAG and reverse, CTCTGTCTCTCTGACCTCACAG.

The $\mathrm{Ct}$ value obtained for the gene of interest was normalized to a housekeeping gene, $G A P D H$, to obtain the $\triangle \mathrm{Ct}$ values. The $\Delta \Delta \mathrm{Ct}$ values were then obtained by subtracting the $\Delta \mathrm{Ct}$ values for each gene of interest from the $\Delta \mathrm{Ct}$ values for the control sample. Results were calculated using the equation $R Q=2^{-\Delta \Delta C t}$, where $R Q$ is the relative quantity, and expressed as the fold change, relative to the gene expression levels in the control samples.

Cell proliferation analysis. MTT (Sigma) and colony-forming assays were used to analyze cell proliferation. Cells were plated in 96 -well plates at $3 \times 10^{3}$ cells per well and cultured for $2,4,6,8$ and 10 days. The cell growth and viability were assessed following incubation in $0.1 \mathrm{mg} / \mathrm{ml} \mathrm{MTT}$ at $37^{\circ} \mathrm{C}$ for $4 \mathrm{~h}$. The cells were lysed in dimethylsulfoxide (Cellgro, Herndon, VA, USA) at room temperature for $10 \mathrm{~min}$. Using a microplate reader (Thermo Fisher Scientific, Inc., Waltham, MA, USA), the absorbance in each well was measured at $492 \mathrm{~nm}$. Colony-forming efficiency was determined by plating the cells at 10 cells $/ \mathrm{cm}^{2}$ in $60-\mathrm{mm}$ dishes (Nunc A/S). After 14 days of culture, dishes were stained with Giemsa and the number of visible colonies was counted. Colony-forming efficiency was calculated as the percentage of the number of cells initially plated that gave rise to visible colonies (>45 cells).

Evaluation of osteogenic differentiation in hBMSCs by ALP staining. ALP staining and analysis were performed after 14 days of incubation in differentiated culture medium. Cells were induced for osteoblast differentiation, washed twice in PBS, fixed in methanol/formalin (9:1; Ziyi, Shanghai, China) for $30 \mathrm{~min}$ and stained with the 5-bromo-4-chloro-3'-indolyphosphate $\mathrm{p}$-toluidine salt/nitro-blue tetrazolium chloride Alkaline Phosphatase Color Development kit (C3206; Beyotime, Haimen, China) according to the manufacturer's instructions. The ALP activity in the monolayers was determined using the p-Nitrophenyl Phosphate Liquid Substrate System (Sigma) according to the manufacturer's instructions. Using a Multiskan Ascent microplate reader (Thermo Fisher Scientific, Inc.), the absorbance was measured at $405 \mathrm{~nm}$.

Mineralization assay in hBMSCs by Alizarin Red staining $(A R S)$. Following being fixed in $4 \%$ paraformaldehyde $(\mathrm{m} / \mathrm{v})$ (Jianglaibio, Shanghai, China) for $10 \mathrm{~min}$, hBMSCs were evaluated by ARS (ScienCell, San Diego, CA, USA) staining. Briefly, the cells were stained with $2 \%$ ARS (pH 4.1) for 15 min and washed twice in PBS. Orange and red staining indicated calcium nodules.

Statistical analysis. Statistical analysis of data was performed using the independent t-test and results were expressed as the mean $\pm \mathrm{SD}$. $\mathrm{P}<0.05$ was considered to indicate a statistically significant difference. All statistical calculations were performed using SPSS software, version 17.0 (SPSS, Inc., Chicago, IL, USA).

\section{Results}

Upregulated expression of miR-125b in osteoporotic hBMSCs. The hBMSCs were isolated from the iliac crest of elderly patients with osteoporosis $(n=4)$ and controls $(n=5)$. The clinical characteristics of each donor are summarized in Table I. Before comparing the expression levels of miR-125b, the proliferation and osteogenic differentiation activity of hBMSCs that were derived from osteoporotic patients and control cases were estimated. The MTT assay was performed on hBMSCs of passage three to estimate the cell proliferation rate. Compared with hBMSCs derived from the control subjects, the hBMSCs derived from osteoporotic patients showed a slower growth rate with a low cell density $(\mathrm{P}<0.05$; Fig. 1A). This result suggested a decreased proliferation rate of osteoporotic hBMSCs.

Upon osteogenic induction, hBMSCs were able to undergo osteogenic differentiation into osteoblasts. The osteogenic differentiation ability of hBMSCs was evaluated by ALP staining. Compared with the control hBMSCs, the senile osteoporotic 
A

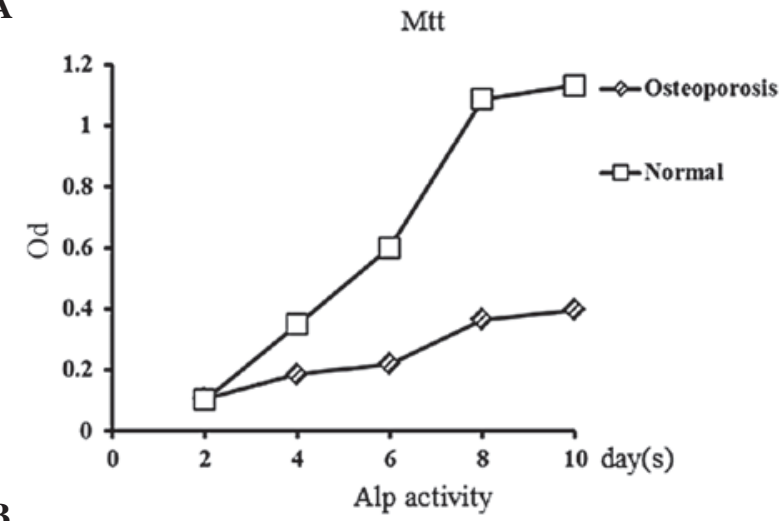

B

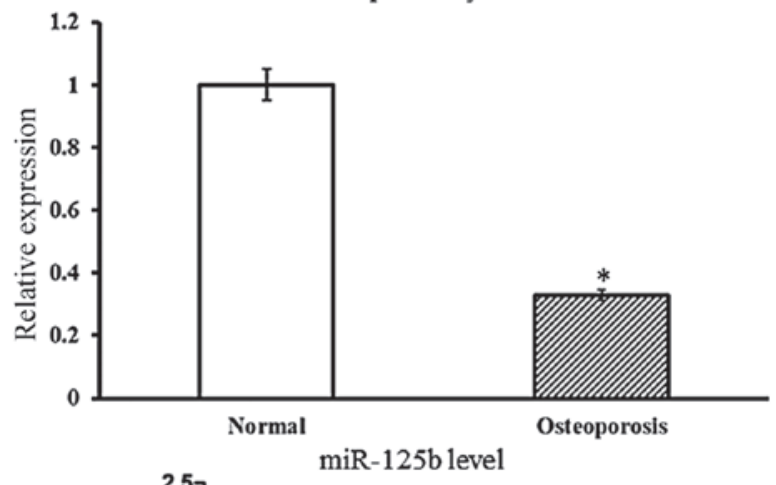

C

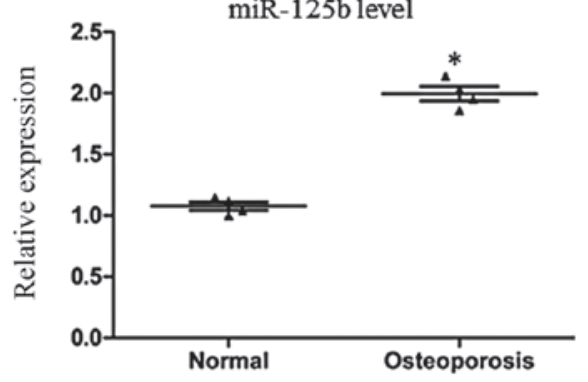

Figure 1. Comparison of the proliferation and osteogenic differentiation of hBMSCs isolated from osteoporotic patients and normal subjects. (A) Growth and viability of hBMSCs were determined by the MTT assay after cells were cultured for 2, 4, 6, 8 and 10 days; (B) hBMSCs at passage 3 were cultured in osteogenic medium for 14 days, followed by staining and assessment of ALP activity. Data were analyzed using the Student's t-tests; (C) quantitative polymerase chain reaction analysis of miR-125b expression in hBMSCs. miR-125b expression levels were increased in hBMSCs isolated from elderly patients. Data were subjected to Student's t-tests. Error bars represent the mean \pm standard deviation of three independent experiments. ${ }^{*} \mathrm{P}<0.05$. hBMSCs, human bone marrow-derived mesenchymal stem cells; ALP, alkaline phosphatase; miR, micro RNA; OD, optical density.

hBMSCs showed decreased ALP activity ( $\mathrm{P}<0.05$; Fig. 1B), which indicated suppression of osteogenic differentiation of osteoporotic hBMSCs.

qPCR was used to estimate the expression levels of miR-125b in osteoporotic hBMSCs as well as the cells derived from the control subjects. Compared to the control hBMSCs, miR-125b expression levels in osteoporotic hBMSCs were significantly upregulated $(\mathrm{P}<0.05$; Fig. 1C).

In conclusion, it was identified that the osteoporotic hBMSCs exhibited decreased cell proliferation and osteogenic differentiation, which correlated with increased expression levels of miR-125b. These results suggested that miR-125b may have a negative impact on cell proliferation or differentiation of hBMSCs; however, this requires validation and further investigation.
A
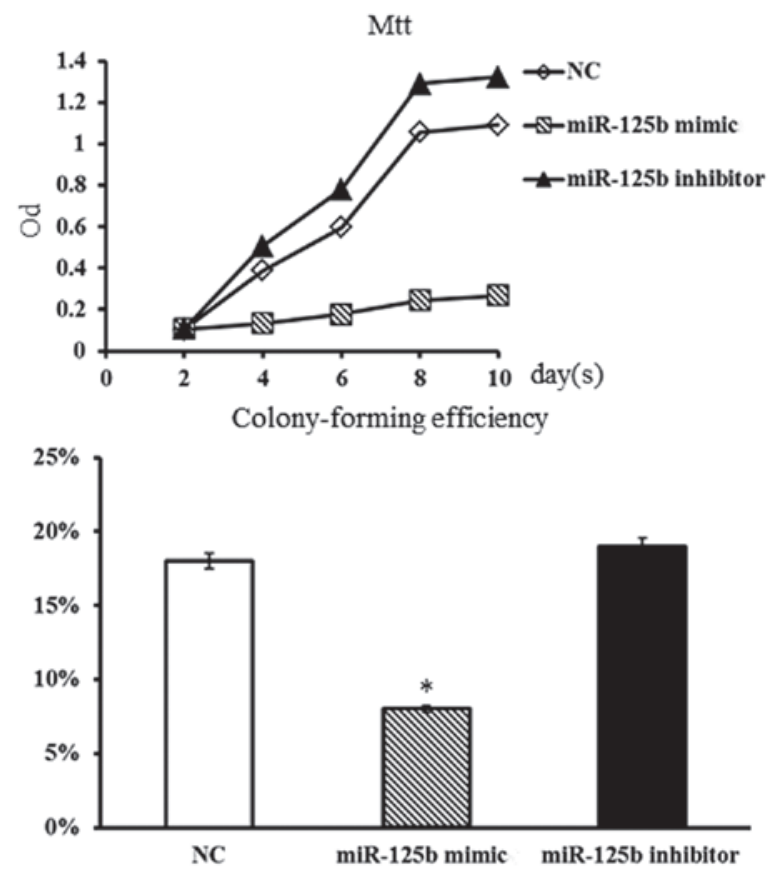

Figure 2. Overexpression of miR-125b suppressed the proliferative ability of hBMSCs. (A) After hBMSCs were transfected with miR-125b mimic or miR-125b inhibitor, cells were cultured for 2, 4, 6, 8 and 10 days, and cell growth and viability were determined using the MTT assay. (B) Colony-forming efficiency was measured after 14 days of culture. Error bar represents the mean \pm standard deviation of three independent experiments. " $\mathrm{P}<0.05$. hBMSCs, human bone marrow-derived mesenchymal stem cells; NC, negative control; miR, micro RNA; OD, optical density.

Impact of miR-125b on cell proliferation in hBMSCs. To study the impact of miR-125b expression on cell proliferation of hBMSCs, a miR-125b mimic and an miR-125b inhibitor were respectively transfected into normal individual hBMSCs. The miR-125b mimic, miR-125b inhibitor and non-specific negative control were designed and synthesized according to the protocol by Liu et al (9). The proliferative rate of hBMSCs was evaluated by MTT and colony-forming assays. Compared with the non-transfected (control) group, hBMSCs transfected with miR-125b inhibitor showed a slightly higher proliferation rate (Fig. 2A). However, the proliferative rate of hBMSCs was markedly suppressed in the presence of the miR-125b mimic. Furthermore, the colony-forming assay also showed consistent results. The colony-forming ability of hBMSCs was obviously decreased in the presence of the miR-125b mimic $(\mathrm{P}<0.05$; Fig. $2 \mathrm{~B})$, while the inhibition of miR-125b in hBMSCs resulted in a slight increase in the number of colonies formed $(\mathrm{P}>0.05)$. These results suggested that the overexpression of miR-125b in normal hBMSCs was able to efficiently suppress the proliferative ability of hBMSCs.

Impact of miR-125b on the osteogenic differentiation in hBMSCs. To study the role of miR-125b expression on osteogenic differentiation in hBMSCs, the miR-125b mimic or the miR-125b inhibitor were transfected into hBMSCs and cultured in osteogenic differentiation medium. After 14 days of culture, the expression of various bone-related genes in hBMSCs was 
A
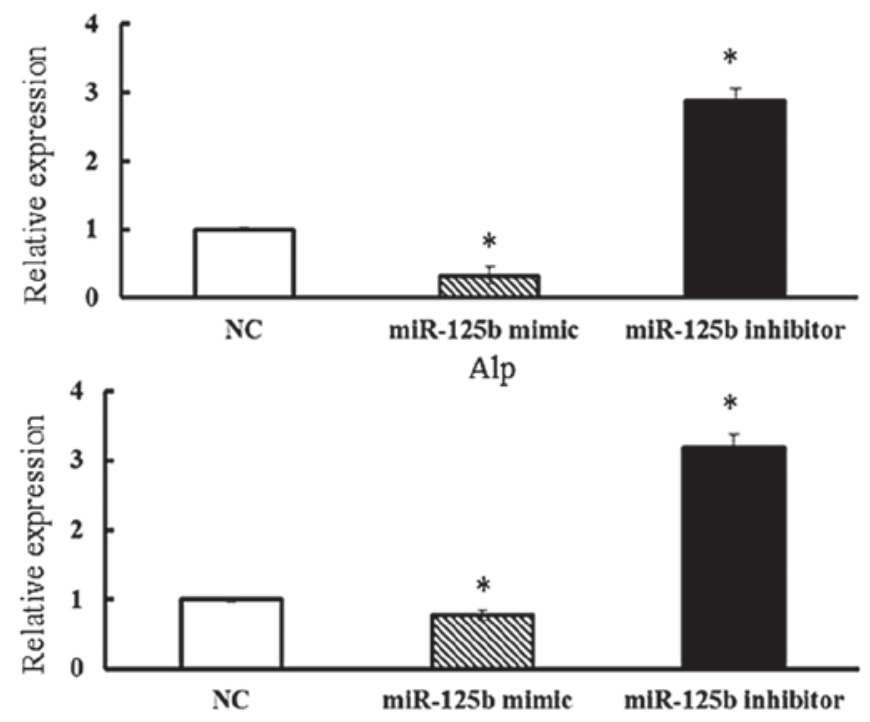

Osteocalcin
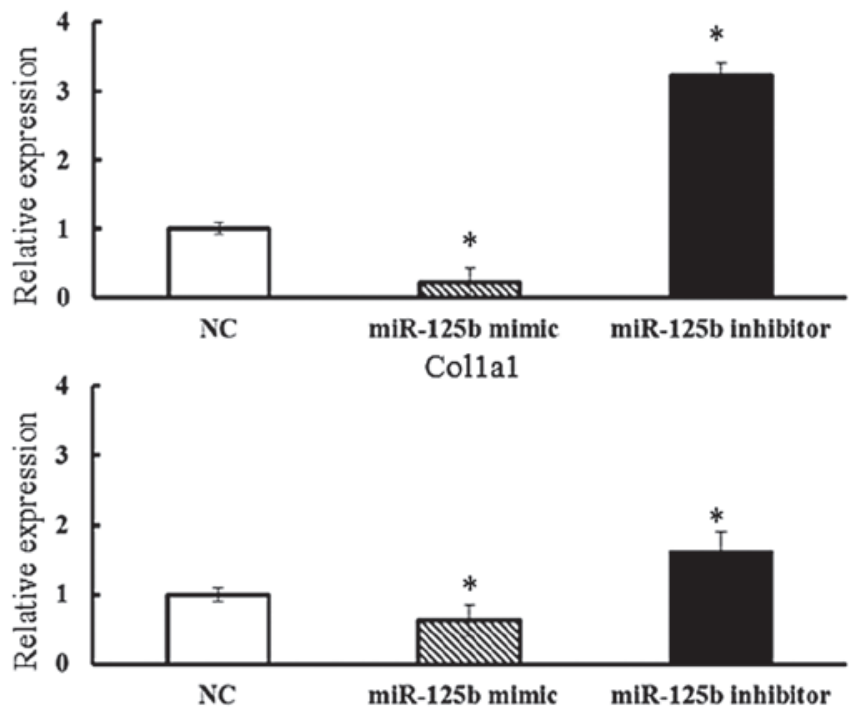

B
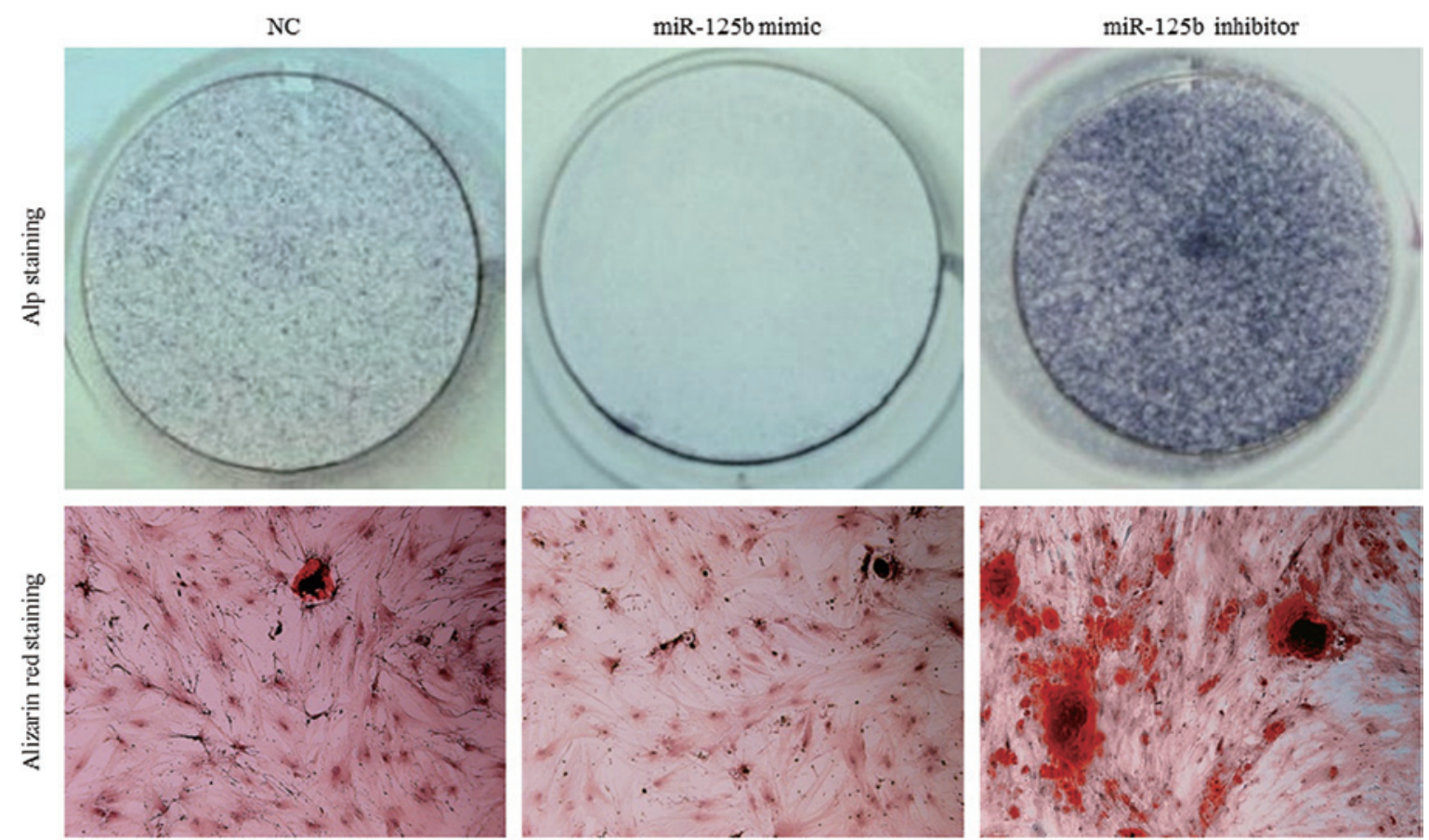

C

Alp activity

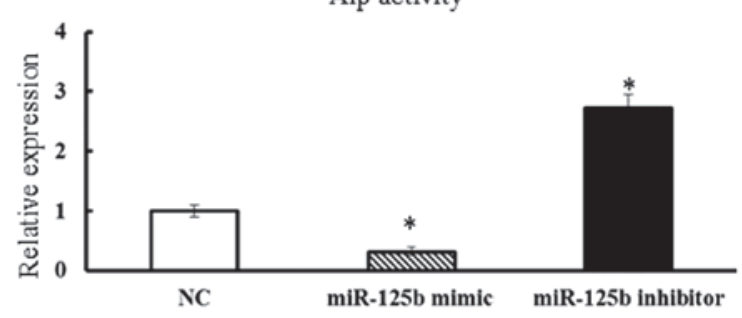

Figure 3. (A) Overexpression of miR-125b suppressed the osteogenic differentiation of hBMSCs. Osteoblast differentiation of hBMSCs was induced by osteogenic differentiation medium and hBMSCs were collected 14 days after osteogenic induction. qPCR analysis measured the expression of Runx-2, $A L P$, $O C$ and COL1 1 in hBMSCs. qPCR data were subjected to Student's t-tests. Error bars represent the mean \pm standard deviation of three independent experiments. ${ }^{\text {"P }}<0.05$. (B) ALP staining was performed after 14 days of culture to detect ALP activity and Alizarin Red staining was performed after 21 days to evaluate mineralized bone matrix formation. (C) ALP activity of hBMSCs was determined 14 days after osteogenic induction by p-Nitrophenyl Phosphate Liquid Substrate System and absorbance was measured at $405 \mathrm{~nm}$. Data were subjected to Student's t-test. "P<0.05. hBMSCs, human bone marrow-derived mesenchymal stem cells; qPCR, quantitative polymerase chain reaction; Runx-2, Runt-related transcription factor-2; ALP, alkaline phosphatase; OC, osteocalcin; COL1 $\alpha 1$, collagen type I $\alpha 1$; NC, negative control; miR, micro RNA; OD, optical density. 
A
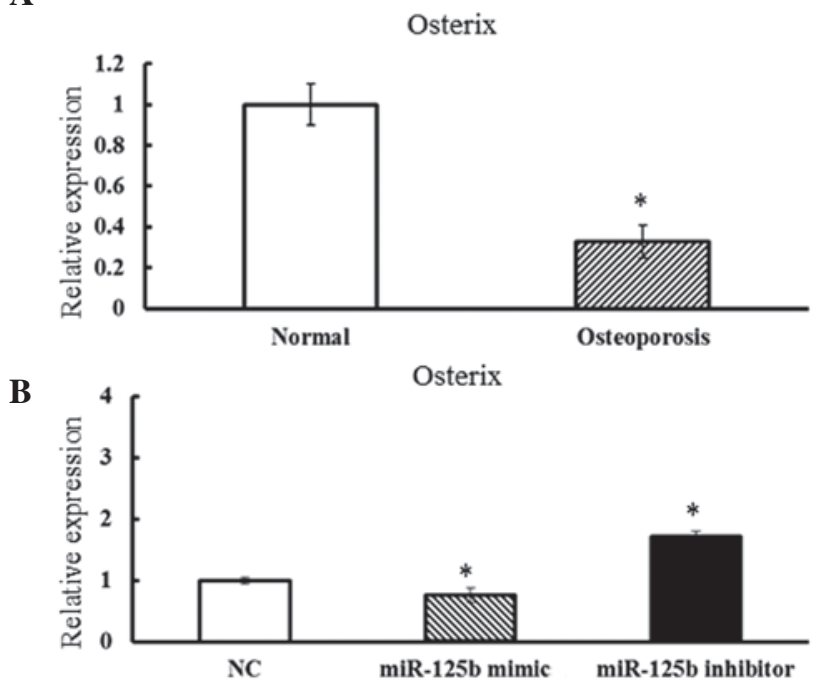

Figure 4. Osterix may function as a mediator of miR-125b on osteogeinic differentiation of hBMSCs. (A) qPCR assay was performed to determine the expression levels of Osx in hBMSCs isolated from elderly osteoporotic patients. (B) qPCR analysis of Osterix expression in hBMSCs after transfection with miR-125b inhibitor or mimic. qPCR data were subjected to Student's t-test. Error bars represent the mean \pm standard deviation of three independent experiments. ${ }^{*} \mathrm{P}<0.05$. hBMSCs, human bone marrow-derived mesenchymal stem cells; qPCR, quantitative polymerase chain reaction.

determined by qPCR. The expression of Runx-2, ALP, COL $1 \alpha 1$ and $O C$ were suppressed in hBMSCs transfected with the miR-125b mimic (Fig. 3A). By contrast, in the presence of miR-125b inhibitors, a significant increase in the expression levels of Runx-2, ALP,OC and COL1al was observed in hBMSCs $(\mathrm{P}<0.05)$ (Fig. 3A).

To further confirm these findings, transfected hBMSCs from each group were subjected to ALP staining on day 14 after culturing in conditioned media. The hBMSCs transfected with the miR-125b mimic showed poor ALP staining, while the hBMSCs transfected with miR-125b inhibitor stained strongly positive for ALP. This was a clear indication of the negative effects of miR-125b expression on osteogenic differentiation of hBMSCs (Fig. 3B and C). The degree of matrix mineralization of hBMSCs was further evaluated by Alizarin red staining on day 21 after transfection (Fig. 3B). Consistent with the results of ALP staining, Alizarin red staining revealed that inhibition of the function of miR-125b in hBMSCs obviously increased matrix mineralization activity (Fig. 3C). In conclusion, the results suggested that miR-125b has a negative regulatory role in osteogenic differentiation processes and the subsequent mineralization of hBMSCs.

Combined effects of Osx and miR-125b expression on osteogenic differentiation of hBMSCs. The possible targets of miR-125b were predicted by online software including (accession date: April 2012): TargetScan (http://www.targetscan. org/) and PicTar (http://pictar.mdc-berlin.de/). Osx, one of the essential transcription factors for the regulation of osteogenic differentiation, was identified as a potential target of miR-125b.

To assess whether $O s x$ was functionally associated with miR-125b, the expression levels of Osx in osteoporotic hBMSCs were assessed. Compared with the cells derived from normal adults, osteoporotic hBMSCs exhibited concurrently lower expression levels of Osx and higher expression levels of miR-125b (Fig. 4A). Furthermore, when the miR-125b mimic was overexpressed in hBMSCs, the expression levels of $O s x$ were dramatically decreased. Furthermore, significantly increased $O s x$ expression levels were observed in hBMSCs transfected with the miR-125b inhibitor (Fig. 4B). The results strongly suggested that $O s x$ may be a target of miR-125b and may mediate the osteogenic differentiation regulated by miR-125b in hBMSCs.

\section{Discussion}

Osteoporosis is characterized by reduced bone mass, which results from an imbalance in bone formation versus resorption (16). The increased activity of osteoclasts has been reported as the main factor involved in the progression of osteoporosis; however, the defects of BMSCs/osteoblasts in osteoporosis have not been fully elucidated. BMSCs, as the main source of adult osteoprogenitors, were found to exhibit age-associated changes in size, changes in proliferative capacity and differentiation potential compared to other MSCs, which suggests that BMSCs have an essential role in the pathogenesis of bone loss (17).

While the regulation of the abnormal proliferation and differentiation of BMSCs that are involved in the pathogenesis of osteoporosis remains to be elucidated, recent studies have shown that certain miRs may have a crucial role in the osteogenic differentiation of BMSCs $(5,18,19)$. However, currently, there are few reports elucidating the roles of miRs in the proliferation and osteogenic differentiation of hBMSCs implicated in the process of senile osteoporosis.

The present study identified that miR-125b acted as a key regulator in the progression of senile osteoporosis. The expression levels of miR-125b in hBMSCs that were derived from patients with osteoporosis and control subjects were assessed. The expression levels of miR-125b were significantly higher in hBMSCs derived from elderly patients compared with those from normal younger subjects.

Furthermore, miR-125b inhibited hBMSC proliferation and osteogenic differentiation. It was identified that $O s x$ was a potential target of miR-125b, contributing to the negative regulatory function of miR-125b in osteogenic differentiation processes in hBMSCs.

miR-125b has been reported to participate in numerous cell activities. The overexpression of miR-125b in SKBR3 cells caused impaired anchorage-dependent growth and reduced migration and invasion capacities by suppressing human epidermal growth factor- 2 and -3 signaling (8). miR-125b is also critical for the suppression of human U251 glioma stem cell proliferation through the cell cycle regulatory proteins CDK6 and CDC25A (20). Furthermore, miR-125b exhibited suppressive effects on the proliferation and migration of osteosarcoma cells by downregulating signal transducer and activator of transcription 3 (9). A recent study reported that miR-125b was downregulated during osteogenic differentiation in human adipose-derived stem cells (21). Moreover, miR-125b was also shown to inhibit osteoblast differentiation of ST2 cells (22). However, in the present study, the molecular mediator of miR-125b on osteogenic differentiation of ST2 cells was not elucidated. Bioinformatics projection and qPCR assays 
identified that Osx (SP7) function may be a potential target of miR-125b and may mediate osteogenic differentiation regulated by miR-125b. Consistent with these results, Goettsch et al (23) indicated that miR-125b expression promoted osteogenic differentiation of human coronary artery smooth muscle cells, targeted by Osx. Osx, which functions downstream of Runx-2/core-binding factor $\alpha 1$, was required for osteoblast differentiation, bone formation (14) and upregulation in the osteogenic differentiation of hBMSCs (24). The present study demonstrated that the endogenous expression of Osx decreased following hBMSC transfection with a miR-125b mimic and increased following transfection with an miR-125b inhibitor.

These results suggested that $O s x$ may be regulated by miR-125b; however, the mechanisms involved in the suppressive effects of miR-125b on the proliferation of hBMSCs requires elucidation. Recent studies have shown that miR-125b expression directly repressed 20 novel targets in the p53 network (25), which had a vital role in the biology of MSCs (26) and regulated osteoblast differentiation via the transcription factor $\operatorname{Osx}(27)$. These studies indicate that miR-125b-dependent regulation of the p53 pathway may account for the negative effects on hBMSCs proliferation. Moreover, there may be additional unknown signaling pathways involved in senile osteoporosis, which may influence the high expression levels of miR-125b.

In conclusion, to the best of our knowledge, the present study was the first to report the roles of miRs in senile osteoporosis. miR-125b may be involved in the progression of senile osteoporosis by suppressing the proliferation and osteogenic differentiation of hBMSCs. Further understanding of the abnormal proliferation and osteogenic differentiation of osteoporotic hBMSCs is required to elucidate the biological characteristics of hBMSCs and also provide a novel clinical treatment for the prevention of osteoporosis.

\section{Acknowledgements}

The present study was supported by the Ministry of Science and Technology of China (no. 2011CB964703), the National Natural Science Foundation of China (no. 30901504) and the China Postdoctoral Science Foundation (nos. 20100480093 and 2012T50856).

\section{References}

1. Pittenger MF, Mackay AM, Beck SC, et al: Multilineage potential of adult human mesenchymal stem cells. Science 284: 143-147, 1999.

2. Tuli R, Tuli S, Nandi S, et al: Characterization of multipotential mesenchymal progenitor cells derived from human trabecular bone. Stem Cells 21: 681-693, 2003.

3. Le Blanc K and Pittenger M: Mesenchymal stem cells: progress toward promise. Cytotherapy 7: 36-45, 2005.

4. Huang J, Zhao L, Xing L and Chen D: MicroRNA-204 regulates Runx 2 protein expression and mesenchymal progenitor cell differentiation. Stem Cells 28: 357-364, 2010.

5. Eskildsen T, Taipaleenmäki H, Stenvang J, et al: MicroRNA-138 regulates osteogenic differentiation of human stromal (mesenchymal) stem cells in vivo. Proc Natl Acad Sci USA 108 6139-6144, 2011.
6. Zhang JF, Fu WM, He ML, et al: MiR-637 maintains the balance between adipocytes and osteoblasts by directly targeting Osterix. Mol Biol Cell 22: 3955-3961, 2011.

7. Willimott S and Wagner SD: MiR-125b and miR-155 contribute to bcl2 repression and proliferation in response to CD40 ligand (CD154) in human leukemic B-cells. J Biol Chem 287: 2608-2617, 2012.

8. Scott GK, Goga A, Bhaumik D, et al: Coordinate suppression of ERBB2 and ERBB3 by enforced expression of micro-RNA miR-125a or miR-125b. J Biol Chem 282: 1479-1486, 2007.

9. Liu LH, Li H, Li JP, et al: miR-125b suppresses the proliferation and migration of osteosarcoma cells through down-regulation of STAT3. Biochem Biophys Res Commun 416: 31-38, 2011.

10. Lin KY, Zhang XJ, Feng DD, et al: miR-125b, a target of CDX2, regulates cell differentiation through repression of the core binding factor in hematopoietic malignancies. J Biol Chem 286: 38253-38263, 2011.

11. Manolagas SC and Jilka RL: Bone marrow, cytokines, and bone remodeling. Emerging insights into the pathophysiology of osteoporosis. N Engl J Med 332: 305-311, 1995.

12. Verma S, Rajaratnam JH, Denton J, et al: Adipocytic proportion of bone marrow is inversely related to bone formation in osteoporosis. J Clin Pathol 55: 693-698, 2002.

13. Yoshida CA, Yamamoto H, Fujita T, et al: Runx2 and Runx3 are essential for chondrocyte maturation, and Runx2 regulates limb growth through induction of Indian hedgehog. Genes Dev 18: 952-963, 2004.

14. Nakashima K, Zhou X, Kunkel G, et al: The novel zinc finger-containing transcription factor osterix is required for osteoblast differentiation and bone formation. Cell 108: 17-29, 2002.

15. Pittenger MF, Mackay AM, Beck SC, et al: Multilineage potential of adult human mesenchymal stem cells. Science 284: 143-147, 1999.

16. Gallagher JC and Sai AJ: Molecular biology of bone remodeling: implications for new therapeutic targets for osteoporosis. Maturitas 65: 301-307, 2010.

17. Bellantuono I, Aldahmash A and Kassem M: Aging of marrow stromal (skeletal) stem cells and their contribution to age-related bone loss. Biochim Biophys Acta 1792: 364-370, 2009.

18. Gao J, Yang T, Han J, et al: MicroRNA expression during osteogenic differentiation of human multipotent mesenchymal stromal cells from bone marrow. J Cell Biochem 112: 1844-1856, 2011.

19. Goff LA, Boucher S, Ricupero CL, et al: Differentiating human multipotent mesenchymal stromal cells regulate microRNAs: prediction of microRNA regulation by PDGF during osteogenesis. Exp Hematol 36: 1354-1369, 2008.

20. Shi L, Zhang J, Pan T, et al: MiR-125b is critical for the suppression of human U251 glioma stem cell proliferation. Brain Res 1312: 120-126, 2010.

21. Zhang ZJ, Zhang H, Kang Y, et al: miRNA expression profile during osteogenic differentiation of human adipose-derived stem cells. J Cell Biochem 113: 888-898, 2012.

22. Mizuno Y, Yagi K and Tokuzawa Y: miR-125b inhibits osteoblastic differentiation by down-regulation of cell proliferation. Biochem Biophys Res Commun 368: 267-272, 2008.

23. Goettsch C, Rauner M, Pacyna N, et al: miR-125b regulates calcification of vascular smooth muscle cells. Am J Pathol 179: 1594-1600, 2011.

24. Liu F, Akiyama Y, Tai S, et al: Changes in the expression of CD106, osteogenic genes, and transcription factors involved in the osteogenic differentiation of human bone marrow mesenchymal stem cells. J Bone Miner Metab 26: 312-320, 2008.

25. Le MTN, Shyh-Chang N, Khaw SL, et al: Conserved regulation of p53 network dosage by microRNA-125b occurs through evolving miRNA-target gene pairs. PLoS Genet 7: e1002242, 2011.

26. Armesilla-Diaz A, Elvira G and Silva A: p53 regulates the proliferation, differentiation and spontaneous transformation of mesenchymal stem cells. Exp Cell Res 315: 3598-3610, 2009.

27. Liu $\mathrm{H}$ and $\mathrm{Li} \mathrm{B}$ : p53 control of bone remodeling. J Cell Biochem 111: 529-534, 2010. 